\title{
12 Ethische Herausforderungen beim Massenanfall von Notfallpatienten
}

\author{
Florian Reifferscheid
}

Der Massenanfall ist dadurch gekennzeichnet, dass eine große Anzahl verletzter oder erkrankter Notfallpatienten die in der prähospitalen Notfallmedizin aktiven Einsatzkräfte an ihre Grenzen führt. Die größte Herausforderung für den an seiner Bewältigung beteiligten Arzt besteht darin, die zumindest in der Anfangsphase knappen Ressourcen so einzusetzen, dass möglichst vielen Patienten geholfen werden kann. Diesem Ziel muss der Wunsch des Arztes nach einer alltagsmedizinisch üblichen individuellen Maximaltherapie geopfert werden.

\subsection{Definition}

Der Massenanfall Verletzter oder Erkrankter (MANV/E) definiert sich nicht über eine starre Anzahl Betroffener oder ein bestimmtes Ausmaß einer Schadenslage. Er resultiert vielmehr aus der Relation des Notfalls zu den vorhandenen Ressourcen.

Dabei kann es sich ebenso gut um einen Verkehrsunfall mit einer größeren Anzahl Verletzter im ländlichen Raum wie um eine Lebensmittelvergiftung in einem Seniorenheim oder einer Schule handeln. Die DIN EN 13050 „Begriffe im Rettungswesen“ (NARK 2009) definiert den MANV/E als „Notfall, mit einer großen Anzahl von Verletzten oder Erkrankten sowie anderen Geschädigten oder Betroffenen“. Abzugrenzen ist er von der Katastrophe, die dieselbe DIN als „über das Großschadensereignis hinausgehendes Ereignis, mit einer wesentlichen Zerstörung oder Schädigung der örtlichen Infrastruktur, das im Rahmen der medizinischen Versorgung mit eigenen Mitteln und Einsatzstrukturen des Rettungsdienstes allein nicht bewältigt werden 
kann“, beschreibt. Im Umkehrschluss kann der MANV/E vom Rettungsdienst allein bewältigt werden.

Die Besonderheit der Katastrophe dagegen besteht nicht allein in ihrem Schadensausmaß, sondern in der Schädigung der Infrastruktur, weshalb ihre Bewältigung erschwert und ihre Dauer deutlich länger ist als die eines MANV/E.

In der Folge der Terroranschläge vom 11. September 2001 und der Hochwasserlagen in Deutschland hat die Konferenz der deutschen Innenminister und -senatoren (IMK) eine „Neue Strategie zum Schutz der Bevölkerung in Deutschland“ (Bundesamt für Bevölkerungsschutz und Katastrophenhilfe 2010) beschlossen und in dieser vier Risikokategorien und korrespondierende Schutz- und Versorgungsstufen beschrieben (vgl. Tab. 19).

\section{Tab. 19 Schutz- und Versorgungsstufen (nach Bundesamt für Bevölkerungsschutz und} Katastrophenhilfe 2010); VS = Versorgungsstufe

\begin{tabular}{|c|c|c|c|}
\hline vS & Beschreibung & Schutzziele & Schutzpotenziale \\
\hline 1 & $\begin{array}{l}\text { Normierter alltäglicher } \\
\text { Schutz }\end{array}$ & $\begin{array}{l}\text { Hilfeleistung für individuelle Notfälle } \\
\text { im Rahmen des Rettungsdienstes }\end{array}$ & $\begin{array}{l}\text { Rettungsdienst gemäß Rettungs- } \\
\text { dienstgesetzen der Länder }\end{array}$ \\
\hline 2 & $\begin{array}{l}\text { Standardisierter, } \\
\text { flächendeckender } \\
\text { Grundschutz }\end{array}$ & $\begin{array}{l}\text { Hilfeleistung für Schadenereignisse } \\
\text { mit einer definierten Zahl Verletzter/ } \\
\text { Erkrankter in einem Zuständigkeits- } \\
\text { bereich, Aufbau und Betrieb von } \\
\text { Patientenablagen }\end{array}$ & $\begin{array}{l}\text { Rettungsdienst, Schnelleinsatz- } \\
\text { gruppen, Teileinheiten des Sanitäts-/ } \\
\text { Betreuungsdienstes des KatS }\end{array}$ \\
\hline 3 & $\begin{array}{l}\text { Erhöhter Schutz für } \\
\text { gefährdete Regionen } \\
\text { und Einrichtungen }\end{array}$ & $\begin{array}{l}\text { Hilfeleistung für Schadenereignisse, } \\
\text { die nicht mit dem Potenzial des Grund- } \\
\text { schutzes abzudecken sind, Aufbau und } \\
\text { Betrieb eines Behandlungsplatzes }\end{array}$ & $\begin{array}{l}\text { Rettungsdienst, Schnelleinsatz- } \\
\text { gruppen, Teileinheiten des Sanitäts-/ } \\
\text { Betreuungsdienstes des KatS, über- } \\
\text { örtliche Hilfe }\end{array}$ \\
\hline 4 & $\begin{array}{l}\text { Sonderschutz mithilfe } \\
\text { von Spezialkräften }\end{array}$ & $\begin{array}{l}\text { Hilfeleistung für Schadenereignisse, die } \\
\text { von Art und Umfang her nicht aus- } \\
\text { schließlich auf der Stufe } 3 \text { bewältigt } \\
\text { werden können, Aufbau und Betrieb } \\
\text { eines Behandlungsplatzes mit der Mög- } \\
\text { lichkeit zur Dekontamination Verletzter }\end{array}$ & $\begin{array}{l}\text { Teileinheiten des Sanitäts-/Betreu- } \\
\text { ungsdienstes des KatS, überörtliche } \\
\text { Hilfe, Medizinische Task Forces, } \\
\text { Analytische Task Forces }\end{array}$ \\
\hline
\end{tabular}

\subsection{Einsatzarten und Besonderheiten}

Immer wieder werden die Rettungskräfte im In- und Ausland mit MANV/E, aber auch Katastrophen konfrontiert. Dabei ist es weniger entscheidend, ob es sich um einen „einfachen“ MANV/E oder eine Katastrophe handelt. Wesentlich ist, wie der Rettungsdienst und die Katastrophenschutzeinrichtungen auf Gefahrensituationen vorbereitet sind und die Einsatzkräfte mit ihnen umzugehen verstehen. Ursachen solcher Einsatzsituationen können so vielfältig sein wie das tägliche Leben. Daher ist die Vorbereitung auf konkrete Situationen nur begrenzt möglich. Ein zentrales Register besteht in Deutschland weder für MANV/E noch für Katastrophen, was die 
wissenschaftliche Beurteilung erschwert. Eine Umfrage (Ellebrecht 2013) unter mehr als 4.ooo Rettungsassistenten und Notärzten hat gezeigt, dass das Gros der MANV/E aus Unfällen mit bis zu 10 Verletzten oder Betroffenen besteht. In diesem Bereich dominieren Verkehrsunfälle. Handelt es sich um Großeinsätze mit mehr als 25 Verletzten oder Betroffenen, so sind dies meist Brände, Gefahrgutunfälle oder Vergiftungen/Erkrankungen. Bei diesen Unfällen sind zwar regelmäßig mehr Personen betroffen, allerdings ist deren Verletzungsgrad geringer als bei Verkehrsunfällen. Hingegen gehen gerade Brände und Gefahrgutunfälle häufig mit Belastungen für die Einsatzkräfte einher, weil es binnen kurzer Zeit die Dynamik der Bedrohung einzuschätzen und einen eventuellen Evakuierungsbedarf festzulegen gilt. Als weitere Auslöser für derartige Konstellationen kommen schwere Unglücksfälle, Naturereignisse und Epidemien in Betracht. Sonderfälle, die den Einsatz spezialisierter Einsatzkräfte erfordern und Helfer und Betroffene mental verunsichern und entsprechend belasten können, sind sogenannte CBRN-Lagen, wozu chemische (C), biologische (B) sowie radiologische (R) und nukleare (N) Gefahren zählen.

Im Bereich der kriminellen oder gar terroristischen Handlungen sehen sich die Helfer mit einer neuen Dimension konfrontiert. Nicht nur müssen sie die willkürlich herbeigeführte Schädigung zahlreicher Unbeteiligter psychisch verarbeiten, besonders belastend sind gerade diejenigen Fälle, bei denen auch die Rettungskräfte durch austretende Gefahrstoffe oder anhaltende kriminelle bzw. terroristische Aktivitäten selbst gefährdet sind und das Ausmaß der Bedrohung nur abstrakt abzuschätzen ist. Vor allem bei Terroranschlägen, das haben Gewaltakte im Nahen Osten, aber auch der Anschlag auf das World Trade Center in New York gezeigt, sind die Rettungskräfte durch auf den Erstschlag folgende weitere Detonationen selbst in Gefahr und damit extremer Beanspruchung ausgesetzt. Ähnlich verhält es sich angesichts von Amoktaten wie etwa in Erfurt oder Winnenden, deren Dynamik und exakte Bedrohung nur schwer einzugrenzen sind.

\subsection{Einsatzkonzepte}

Die Bewältigung eines größeren Notfallereignisses bedarf eines Einsatzkonzeptes, das bereits von den ersten an der Schadensstelle eintreffenden Kräften angewandt und gebahnt wird. Gerade die Initialphase eines solchen Einsatzes hat einen maßgeblichen Einfluss auf den weiteren Verlauf und den Erfolg des Einsatzes. Bisher gibt es kein bundesweit einheitliches und allgemein verbindliches Konzept, allerdings gibt es eine Reihe von Einzelschritten, die zur Bewältigung eines größeren Notfallereignisses erforderlich sind. Nach der allgemein notwendigen Sorge um einen ausreichenden Eigenschutz der Einsatzkräfte gilt es, einen eventuell vor Ort befindlichen - ggf. vorläufigen - Einsatzleiter aufzusuchen und sich mit diesem über die Lage und die veranlassten Maßnahmen abzusprechen. Dieser kann, je nach Einsatzart, der ranghöchste anwesende Mitarbeiter der Feuerwehr, der Polizei oder des ersteintreffenden Rettungsmittels des Rettungsdienstes sein. Im Anschluss daran muss eine rasche Lageerkundung durchgeführt werden. Deren Ziel ist es, das Ausmaß des Schadens, die Anzahl Geschädigter und deren Verletzungs- oder Erkrankungsschwere, eventuelle Gefahrenquellen (bspw. auslaufende Betriebsstoffe, Feuer, Gase, Elektrizität, etc.), Art und Umfang der zur Bewältigung erforderlichen Kräfte sowie eine räumliche Orientierung für die nachfolgende Strukturierung der Einsatzstelle abzu- 
schätzen. Mit den Ergebnissen der Lageerkundung muss eine qualifizierte Rückmeldung an die einsatzführende Leitstelle erfolgen. In dieser sind darüber hinaus Anfahrmöglichkeiten und Bereitstellungsräume sowie der Funkrufname des Ansprechpartners vor Ort zu nennen.

\begin{abstract}
Diesen ersten Schritten kommt eine sehr hohe Bedeutung zu. Es ist essenziell, dass die organisatorischen Maßnahmen hinsichtlich der Struktur der Einsatzstelle und ihrer Umgebung in jedem Fall der individualmedizinischen Versorgung der Patienten vorausgehen.
\end{abstract}

Gerade Fehler in der Raumordnung können im weiteren Verlauf des Einsatzes nicht korrigiert bzw. ausgeglichen werden. Der Umstand, dass gerade in strukturschwachen, ländlichen Einsatzgebieten die häufig kleine Zahl an Einsatzkräften auf absehbare Zeit nicht mit der Patientenversorgung, sondern mit planerischen Aufgaben betraut ist, stellt für diese Helfer regelmäßig ein Dilemma dar. Was die Patienten angeht, müssen erste Patientenablagen definiert werden. Diese dienen einerseits als Übergabepunkte, wenn Patienten von Feuerwehr oder Polizei aus dem Gefahrenbereich gerettet werden müssen, und andererseits der Konzentration an festgelegten Orten, um deren weitere Versorgung übersichtlicher zu strukturieren. Gegenstand der Diskussion ist es, ob man bereits an dieser Stelle Unverletzte bzw. leicht von schwerer Verletzten trennen und ggf. an einem weiter entfernten Ort sammeln sollte.

\title{
12.4 MTF und weitere Katastrophenschutzeinheiten
}

Auch wenn die DIN 13050 den MANV/E definitiv von der Katastrophe unterscheidet, da Ersterer mit den eigenen Kräften und Mitteln des Rettungsdienstes zu bewältigen sei, kommt es in der Praxis bereits bei niedrigeren Schwellen zur Vermischung der jeweiligen Einheiten (entsprechend Versorgungsstufe 2). So ist gerade im ländlichen Raum bei größeren Notfallereignissen häufig der Einsatz sogenannter Schnelleinsatzgruppen (SEG) geboten. Diese rekrutieren sich meist aus ehrenamtlichen Helfern der fünf Hilfsorganisationen oder aus dienstfreien Kräften des regulären Rettungsdienstes. SEG sind vielfach Teileinheiten von Katastrophenschutzformationen etwa der Einsatzeinheiten des Deutschen Roten Kreuzes (DRK), sodass hier ein fließender Übergang vom Einsatz der regulären Rettungsdienstkräfte hin zu dem der Katastrophenschutzeinheiten erfolgt. Gerade wenn es sich bei einem MANV/E um einen länger dauernden Einsatz, bspw. infolge eines Feuers oder einer Evakuierung, handelt, werden neben den reinen sanitätsdienstlichen auch betreuungdienstliche Einheiten zum Einsatz gebracht. Vom Bund werden aktuell über ganz Deutschland verteilt 61 sogenannte Medical Task Forces (MTF) (Bundesamt für Bevölkerungsschutz und Katastrophenhilfe 2015) aufgebaut. Diese Komponenten sind vornehmlich für den überregionalen Einsatz bei großflächigen Katastrophen wie etwa dem regelmäßig wiederkehrenden Elbehochwasser konzipiert und so ausgerichtet, dass sie mindestens 48 Stunden autark arbeiten können. Dabei sollen die Kräfte der MTF entweder an der Schadensstelle bspw. Behandlungsplätze (BHP) einrichten und betreiben, um eine Versorgung vor Ort zu bewerkstelligen, oder bei zerstörter Infrastruktur, mit der Einrichtung eines BHP vor einem Krankenhaus auch örtliche Strukturen der Gesund- 
heitsversorgung verstärken. Den Hilfsorganisationen, die die Einsatzkräfte für die MTF stellen und so deren Einsatzbereitschaft aufrechterhalten, sind deren Fahrzeuge und Mittel ausdrücklich auch zu organisationseigenen oder Zwecken des erweiterten Rettungsdienstes überlassen.

\subsection{Hilfe nach dem Maß der Not - die Sichtung}

Unabhängig davon, welche Kräfte letztendlich zum Einsatz kommen, ist bei MANV/E und Katastrophen gerade zu Beginn ein häufig eklatantes Missverhältnis zwischen Opfern und Helfern anzutreffen. Dieses Missverhältnis, geprägt von wenigen Rettungskräften, die zudem zunächst organisatorische Maßnahmen ergreifen müssen, begründet auch die größte ethische Herausforderung in der Bewältigung einer solchen Einsatzsituation. Notarzt und Notfallsanitäter müssen entscheiden, in welcher Abfolge und welchem Ausmaß Betroffenen geholfen werden kann. Diese notwendige Festlegung einer Reihenfolge der zu Versorgenden ist auch als Triage bekannt. Der Ausdruck ist vom französischen Verb trier für sortieren abgeleitet und wurde während der napoleonischen Kriege geprägt (Gerlach 2012), als es zu entscheiden galt, welcher verwundete Soldat eine Chance auf Heilung bekam und welcher auf dem Schlachtfeld zurückgelassen werden musste. Um die militärische Konnotation zu vermeiden, wird im zivilen Kontext der Begriff Sichtung vorgezogen.

Ziel der Sichtung in der Katastrophenmedizin ist eine möglichst lange Aufrechterhaltung bzw. möglichst schnelle Wiederherstellung individualmedizinischer Versorgungsstrukturen für eine Vielzahl Geschädigter (Sefrin u. Weidringer 2003).

In der Absicht, die bestehenden Vereinbarungen zur Sichtung zu vereinheitlichen und zu vereinfachen, lud die Schutzkommission beim Bundesinnenminister 2002 zahlreiche notfall- und katastrophenmedizinische Experten aus Deutschland und dem benachbarten Ausland zur Beratung ein. In dieser Konferenz wurden vier Sichtungskategorien definiert, die sowohl beim MANV/E als auch in Katastrophenfällen zur Anwendung kommen sollen. Darüber hinaus wurde ein Mindestdatensatz zur Sichtungsdokumentation (s. Tab. 20) beschlossen.

\section{Tab. 20 Mindestdatensatz zur Sichtungsdokumentation (nach Sefrin u. Weidringer 2003)}

\begin{tabular}{ll}
\hline Patientennummer & Unverwechselbare Identifikationsnummer \\
\hline Sichtungskategorie in römischen Zahlen & farbcodiert nach Ampel-Schema \\
\hline Kurzdiagnose & einschließlich „Strichmännchen“ \\
\hline
\end{tabular}

Teil des hippokratischen Eids ist es, jeden Patienten ohne Ansehen von Alter, Konfession, ethnischer Herkunft, Geschlecht, Staatsangehörigkeit oder sozialer Stellung gleich zu behandeln. Eine die medizinische Behandlung priorisierende Sichtung darf, diesen ethischen Grundsatz beachtend, allein von der Bewertung der Schädigungsschwere geleitet sein. Ziel einer solchen Sichtung muss es sein, akut lebensbedrohlich gefährdete Patienten, die einer sofortigen Behandlung oder eines zügigen Trans- 
ports bedürfen, $z u$ identifizieren und sie von denjenigen zu trennen, die nicht oder nur leicht verletzt sind (Adler et al. 2011). Im Rahmen der Sichtung müssen die therapeutischen auf lebensrettende Sofortmaßnahmen (LSM) wie das Freimachen der Atemwege, das Stillen einer stark blutenden Wunde oder die stabile Seitenlage begrenzt werden. Gemäß der Konsensuskonferenz (Sefrin u. Weidringer 2003) werden akut vital bedrohte Patienten, die einer sofortigen Behandlung bedürfen, der Sichtungskategorie 1 (rot) zugewiesen. Schwer verletzte oder erkrankte Patienten, bei denen keine vitale Bedrohung vorliegt, werden der Sichtungskategorie 2 (gelb) und solche, bei denen eine spätere, möglicherweise ambulante Behandlung ausreichend erscheint, der Sichtungskategorie 3 (grün) zugeteilt (s. Tab. 21). Die Verwendung der Kategorie 4 (blau) für Patienten, deren Schädigung so gravierend ist, dass sie unter den Umständen begrenzter Ressourcen ohne Überlebenschance sind, wird nach wie vor kontrovers diskutiert. Einigkeit besteht weitgehend darin, dass diese Kategorie nur zur Anwendung kommen soll, wenn die Merkmale einer Katastrophe und hier vor allem die zerstörte oder beschädigte Infrastruktur, gegeben sind. Gleichwohl setzt der Vollzug der Sichtung den dafür Verantwortlichen, der ihr Patienten, denen im Falle eines Individualnotfalls und einer individualmedizinischen Maximalversorgung eine Rettung möglicherweise zuteil würde, zuzuweisen hat, ethisch gewaltig unter Druck. Die Entscheidung, Patienten eine Behandlung vorzuenthalten, bis die Ressourcen an der Einsatzstelle ausreichen, um damit zu beginnen, erhöht die Wahrscheinlichkeit ihres Versterbens. Die Frage nach ihrer Zulässigkeit zur möglichen Rettung mehrerer weniger schwer geschädigter Patienten kann nur für den jeweiligen konkreten Fall bewertet und nicht abschließend beantwortet werden. Moralisch muss solchen Patienten auch in der Katastrophe eine angemessene, palliative, ärztliche Behandlung zuteil werden, damit ihnen zumindest Schmerzen genommen werden können. Tote werden entsprechend, oftmals schwarz, gekennzeichnet. Sie sollten von den übrigen Patienten separiert werden, auch wenn dies die unumgängliche, nachfolgende Arbeit der Ermittlungsbehörden erschwert.

Tab. 21 Sichtungskategorien und ihre Behandlungskonsequenzen (nach Sefrin u. Weidringer 2003)

\begin{tabular}{|llll}
\hline Farbe & Sichtungskategorie & Beschreibung & Konsequenz \\
\hline Rot & 1 & Akute, vitale Bedrohung & Sofortbehandlung \\
\hline Gelb & 2 & Schwer verletzt/erkrankt & Aufgeschobene Behandlungsdringlichkeit \\
\hline Grün & 3 & Leicht verletzt/erkrankt & Spätere (ambulante) Behandlung \\
\hline Blau & 4 & Ohne Überlebenschance & Betreuende (abwartende) Behandlung \\
\hline & & Tote & Kennzeichnung \\
\hline
\end{tabular}

Die Sichtung selbst ist als dynamischer Prozess zu betrachten und muss wiederholt durchgeführt werden. Die frühere Absicht, bereits mit der ersten Sichtung zugleich eine Entscheidung über Transportprioritäten $z u$ treffen, wurde 2002 verlassen. So beschreibt bspw. Sefrin (2012) hinsichtlich der Besonderheiten des jeweiligen Sichtungsvorgangs drei verschiedene Typen der Sichtung:

1. Vorsichtung - im unmittelbaren Schadensbereich, um zu entscheiden, wer zuerst gerettet bzw. einer interventionellen Therapie zugeführt werden muss. 
2. Behandlungssichtung - nach Einrichtung einer Behandlungsstruktur, um die Patienten den jeweiligen Behandlungsmöglichkeiten zuzuweisen, bspw. am Eingang eines BHP.

3. Transportsichtung - nach dem Erkennen der Behandlungsgrenzen oder der Stabilisierung des Patienten vor Ort, um die Reihenfolge des Abtransports festzulegen.

\subsection{Vorsichtung}

Da gerade im ländlichen Raum die Ressource Notarzt über einen längeren Zeitraum zu Beginn der Schadensbewältigung knapp ist, hat sich eine 2012 nachfolgende Sichtungskonferenz der Schutzkommission für die Durchführung einer Vorsichtung durch Rettungsassistenten bzw. Notfallsanitäter ausgesprochen. Diese Sichtung wird bereits von den ersten eintreffenden Rettungskräften begonnen und nicht bis zum Eintreffen des ersten oder gar des Leitenden Notarztes verzögert. Zu diesem Zweck haben sich verschiedene Algorithmen etabliert, mit denen vor allem die von einem Notfallereignis schwer Betroffenen identifiziert werden können. Eine einfache Option ist die sogenannte Sweeping Triage, bei der Patienten rot und nicht rot gekennzeichnet werden. Von den Handreichungen für eine Vorsichtung ist der mSTaRT-Algorithmus (Kanz et al. 20o6; Offterdinger et al. 2014), eine Modifikation des in den USA gebräuchlichen STaRT-Algorithmus (STaRT = Simple Triage and Rapid Treatment), bei der neben der reinen Sichtungsentscheidung auch erste therapeutische Maßnahmen beschrieben sind, in Deutschland am weitesten verbreitet. Bedenkt man, dass für die Sichtung eines Patienten bis zu zwei Minuten angesetzt werden müssen (Gerlach 2012), so wäre ein einzelner Notarzt mit dieser Aufgabe womöglich unverhältnismäßig lange gebunden und von ersten notfallmedizinischen Interventionen abgehalten.

Es ist also sinnvoll, gerade bei knappen Ressourcen, die Vorsichtung zunächst auf nicht ärztliches Personal und gegebenenfalls mehrere Schultern zu verteilen, um einen schnellen Überblick darüber zu gewinnen, wie schwer die Opfer beeinträchtigt sind und die als rot gesichteten Patienten frühzeitig aus dem Schadensgebiet retten zu können.

Da keiner der vorhandenen Algorithmen wissenschaftlich evaluiert ist, hat die Schutzkommission die Entwicklung und Evaluation eines Algorithmus in Auftrag gegeben. Entwickelt wurde in der Folge der PRIOR-Algorithmus (Primäres Ranking zur initialen Orientierung im Rettungsdienst) (s. Abb. 7), der im Gegensatz zu anderen sich sowohl für chirurgische als auch für konservativ-medizinische Beeinträchtigungen eignet (Bubser et al. 2014). PRIOR orientiert sich am rettungsdienstlich inzwischen etablierten ABCDE-Schema und beginnt mit der Frage nach einer CBRNLage. In der Folge werden die wichtigsten Vitalfunktionen abgefragt und entsprechend pathologischer Zustände bewertet, um die Sichtungskategorien 1-3 zu vergeben. Die Autoren waren bemüht, mit diesem Algorithmus auch bspw. kardial bedrohte Patienten bei einem Feuer zu identifizieren und sie nicht aufgrund ihrer Geh- 


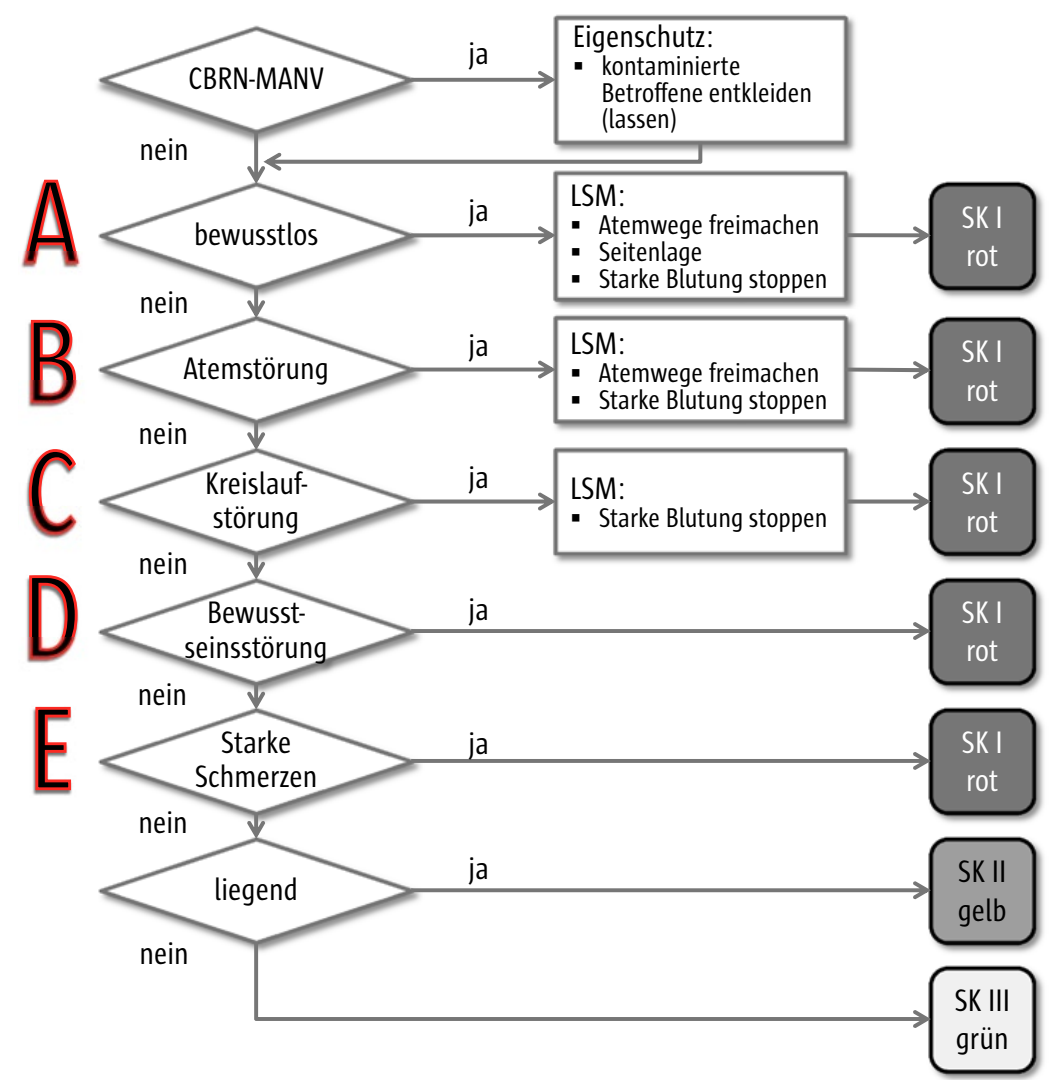

\section{Abb. 7 PRIOR-Algorithmus (nach Bubser et al. 2014)}

fähigkeit wie bspw. beim mSTaRT zunächst in die Kategorie $3 \mathrm{zu}$ sichten. Die Verwendung eines möglichst einheitlichen Algorithmus für eine Erst-, aber auch für folgende Sichtungen durch nicht ärztliches oder ärztliches Personal gibt den Helfern eine Orientierung und erleichtert ihnen nicht nur die Einteilung in die jeweilige Sichtungskategorie, sondern zugleich den Umgang mit diesen Entscheidungen nach Abschluss des Einsatzes bei dessen persönlicher Bewältigung.

Die Erfahrung aus vergangenen Großschadensfällen zeigt, wie wichtig es ist, dass nicht nur jeder Patient im Laufe eines solchen Einsatzes obligat mindestens einmal ärztlich gesichtet werden muss, sondern dass vor allem die für tot erklärten Opfer vor einem Abtransport von der Einsatzstelle noch einmal von einem Arzt untersucht werden müssen. Die Feststellung des Todes bleibt auch beim MANV/E ein ärztliches Primat. 


\subsection{Dokumentation}

Zur Dokumentation des Sichtungsergebnisses hat die Konsensuskonferenz einen Mindestdatensatz definiert (s. Tab. 20). Dieser ist Bestandteil der meisten sogenannten Verletztenanhängekarten, mit denen Betroffene gekennzeichnet werden können. Leider gibt es in Deutschland derzeit kein einheitliches und flächendeckend verbindliches System, weshalb die Vielzahl verschiedener solcher Anhängekarten gerade die Arbeit bei überregionalen MANV/E erschwert. Exemplarisch sei hier die Karte des DRK (s. Abb. 8) genannt, die vermutlich allein aufgrund der Cröße und Aktivität des DRK in diesem Bereich die weiteste Verbreitung hat. Das System ist geeignet, Informationen zu Patient und Sichtungsergebnis zuverlässig zu erfassen und auch deren Entwicklung festzuhalten. Daneben kann das Sichtungsergebnis deutlich sichtbar farbcodiert und bei Veränderungen auch aktualisiert werden. Die DRK-Karte enthält zudem eine Suchdienstkarte und ist damit bei länger anhaltenden Katastrophensituationen ohne Veränderungen ebenfalls anwendbar. Daneben ist es wichtig, an den einzelnen Stationen eine Übersichtsdokumentation zu führen, damit sichergestellt ist, dass jeder Beteiligte erfasst und über seinen Verbleib ein Nachweis geführt wurde.

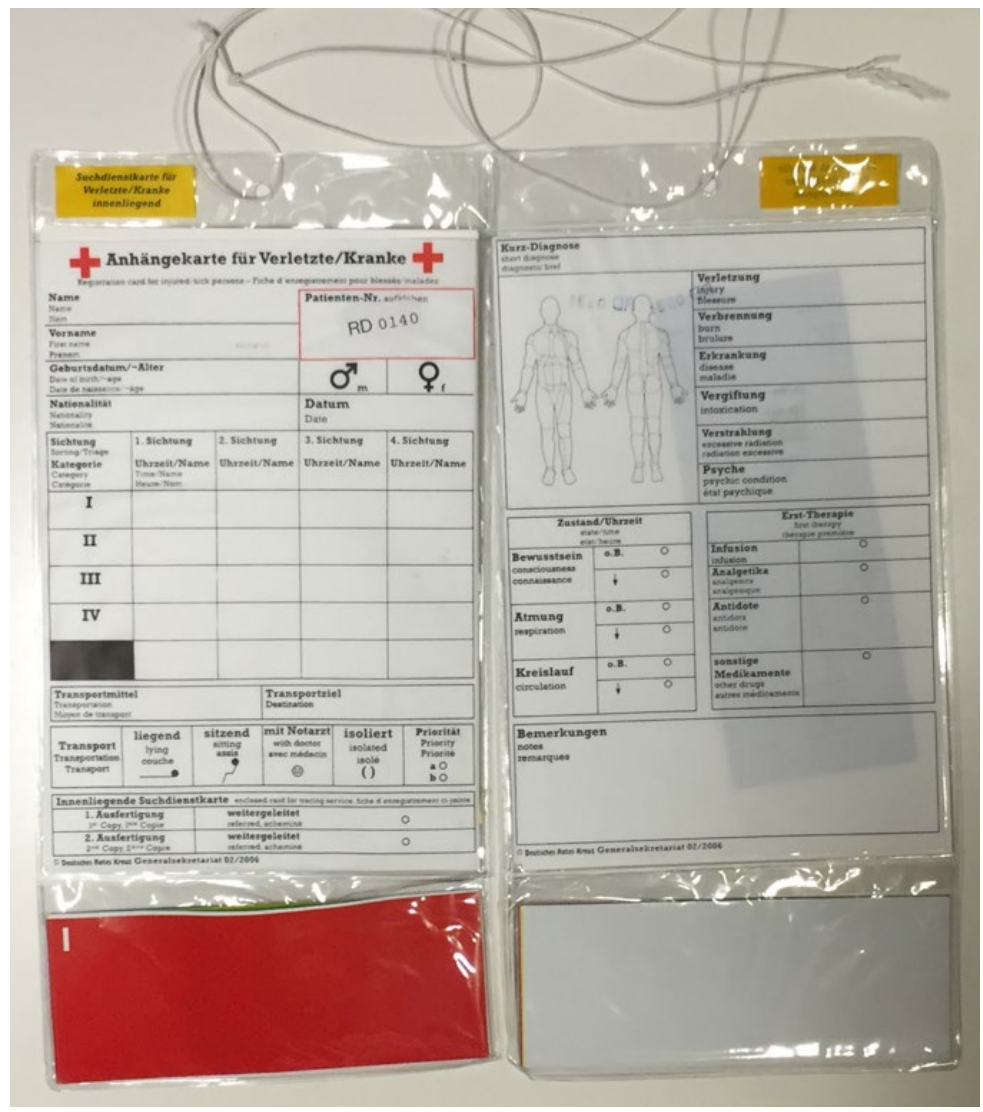

Abb. 8 Verletztenanhängekarte des DRK 


\subsection{Krisenintervention und Notfallseelsorge}

Bei einem MANV/E kommt der Krisenintervention und Notfallseelsorge bei der Versorgung von Betroffenen an der Einsatzstelle eine besondere Bedeutung zu. Der frühzeitige Einsatz entsprechender Kräfte zur Betreuung und Information der leicht oder unverletzten wie auch der, so weit angewandt, in die Kategorie 4 gesichteten Patienten kann die medizinischen Einsatzkräfte entlasten und den Betroffenen eine wichtige Hilfe sein. Im Nachgang eines entsprechenden größeren Notfallereignisses spielen Krisenintervention bzw. entsprechend spezialisierte Teams auch in der Begleitung der Einsatzkräfte eine wichtige Rolle, um posttraumatische Belastungsstörungen oder andere unter dem Begriff des „Second Victim“ (Schiechtl et al. 2013) zusammengefasste Gesundheitsschäden bei den Helfern zu vermeiden.

\subsection{Training und Vorbereitung}

Jeder MANV/E und jede Katastrophensituation sind anders. Daher sind, abgesehen von wenigen Ausnahmen, die Vorbereitung und das Training auf spezielle Situationen schwierig. Allerdings kommt es bei der Bewältigung jedes größeren Notfallereignisses auf die Beachtung der immer gleichen wesentlichen, insbesondere organisatorischen Schritte an. Daher ist es wichtig, dass Erfahrungen, sei es aus Übungen oder realen Einsätzen, geteilt und die Lehren daraus für alle gezogen werden können. Regelmäßige theoretische, vor allem aber praktische Fortbildungen und Übungen, bspw. anhand von Planspielen, sind wertvolle und obligate Bestandteile der Vorbereitung. Sie helfen im Ernstfall den Handelnden, die notwendigen Entscheidungen auf eine breitere Basis zu gründen. Dabei ist es sinnvoll, alle infrage kommenden Einsatzgruppen, Rettungsfachpersonal, Notärzte und Angehörige der Feuerwehren und Polizei, eines Gebietes möglichst gemeinsam trainieren zu lassen, damit die Abläufe im Ernstfall reibungsloser vonstatten gehen. Ein nationales MANV/E-Register könnte einen wertvollen Beitrag leisten, Besonderheiten verschiedener Szenarien herauszuarbeiten und die Vorbereitung entsprechend zu optimieren.

\section{Literatur}

Adler C, Donner A, Kühling M, Krüsmann C (2011) (Vor-)Sichtung und elektronische Betroffenenerfassung bei einem Massenanfall von Verletzten. In: Mendel K, Hennes P (Hrsg.) Handbuch des Rettungswesens. Mendel Verlag, Witten

Bubser F, Callies A, Schreiber |, Grüneisen U (2014) PRIOR: Vorsichtungssystem für Rettungsassistenten und Notfallsanitäter. Rettungsdienst 37(8): 730-4

Bundesamt für Bevölkerungsschutz und Katastrophenhilfe (2010) Neue Strategie zum Schutz der Bevölkerung in Deutschland. Wissenschaftsforum Band 4. 2010

Bundesamt für Bevölkerungsschutz und Katastrophenhilfe (2015) Das neue Ausstattungskonzept des Bundes - Die Medizinische Task Force (MTF). http://www.bbk.bund.de/SharedDocs/Downloads/BBK/DE/MTF/ AlggemeineGrundlagen/Ausstattungskonzept_2007.html

DIN-Normenausschuss Rettungsdienst und Krankenhaus (NARK) (2009) DIN 13050 Begriffe im Rettungswesen

Ellebrecht N (2013) Die Realität der Sichtung. Notfall Rettungsmed. 16(5): 369-76

Gerlach K (2012) Präklinische Triage-Systeme. Notfallmedizin up2date 7: 181-97

Kanz H, Hornburger P, Kay M, Mutschler W, Schäuble W (2006) mSTaRT-Algorithmus für Sichtung, Behandlung und Transport bei einem Massenanfall von Verletzten. Notfall Rettungsmed 9: 264-70 


\subsection{Training und Vorbereitung}

Offterdinger M, Ladehof K, Paul A, Hansen M (2014) Eine einfache Checkliste als Hilfsmittel zur Vorsichtung mit dem mSTaRT-Algorithmus. Notfall Rettungsmed. 17: 415-9

Schiechtl B, Hunger M, Schwappach D, Schmidt C, Padosch S (2013) „Second victim“. Anaesthesist 62: 734-41

Sefrin P (2012) Sichtung - zentrales Element zur Bewältigung eines Großschadensfalls und einer Katastrophe. Der Notarzt 28: 194-202

Sefrin P, Weidringer JW (2003) Sichtungskategorien und deren Dokumentation. Dtsch Ärztebl. 100: 2057-8

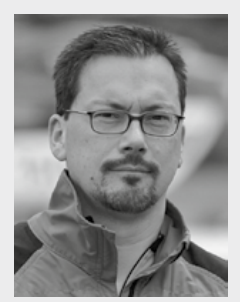

\section{Dr. med. Florian Reifferscheid}

Facharzt für Anästhesiologie und Notfallmedizin. Nach dem Studium an der Universität Hamburg, mit Auslandsaufenthalt in der Schweiz, erfolgte die Facharztausbildung an der Klinik für Anästhesiologie und Operative Intensivmedizin des Universitätsklinikums Schleswig-Holstein, Campus Kiel. Ärztlicher Leiter Notarztdienst der Klinik und leitender Hubschrauberarzt des Christoph 42 in Rendsburg sowie Sprecher der Leitenden Notarztgruppe des Kreises Rendsburg-Eckernförde. Engagement als Landesarzt des DRK-Landesverbands Hamburg und in den Vorständen der Arbeitsgemeinschaft in Norddeutschland tätiger Notärzte (AGNN) und des Forums Leitender Notärzte in Schleswig-Holstein (FLN-SH). 